\title{
MANAGEMENT OF A BURNT PATIENT
}

\author{
by Dennis Walker, F.R.C.S., Plastic Surgeon, Johannesburg General Hospital.
}

Treatment Notes by Marjorie Humphrey, B.Sc. (Phys.)

T here is no such thing as "a treatment for'burns". Damage by heat to the largest organ of the body, the skin, is the start of a series of illnesses whose duration, severity and outcome are frequently in doubt and demand considerable judgment, with laboratory help, for their treatment.

There was a time when descriptions of burn dressings were different in almost every text book one consulted and new applications and dressing routines appeared in Medical Journals practically every month. This no longer holds; the most radical change in our approach to the burnt patient may be summarised in the realisation that a severe burn is a problem in altered blood chemistry. The patient does not usually die because of what is done to the burnt area but frequently because changes are ignored in the patient as a whole. If these changes can be steered back towards a normal bio-chemical picture chances of survival are immeasurably improved.

One of the greatest pitfalls of modern Medicine is to treat the disease and not the patient-we can avoid it, in severly burnt patients, by studying some componants of the series of illnesses which characterise the weeks, and even, months which follow the tragic few moments which turn an active citizen into a burnt patient.

Burns are caused, as a rule, by heat, but the same basic changes may be produced by severe cold. Scalds, usually described as being caused by moist heat, do not differ essentially from the damage by dry heat.

What makes some burns more dangerous than others?

There are several answers to this question:-

1. The Area of the burn is the most important single factor.

2. Depth is usually a secondary consideration. This may be shown by the example of a red hot wire or thin metal rod at high temperature which, in a factory accident, may pass right through a hand. The depth of the burn is greatinvolving all structures present-skin, subcutaneous fat, tendons, blood vessels, muscle and possibly bone-but one would not expect this burn to be fatal. On the other hand a small baby accidentally dropped into a bath of boiling water and instantly rescued may receive a burn' which does not involve the full thickness of the skin, let alone deep structures, but death from this accident is common-the extensive area is the killing factor.

3. Age plays an important part when people at the extremes of life are badly burnt-as with many other injuries, the very young and the very old suffer severly. The former because their surface area and fiuid balance bear one another an easily disturbed relationship-rapidly threatening life, and almost equally dangerous if carelessly and over-enthusiastically remedied-"drowning" by over treatment with intravenous fluids is not unknown. The healthy young adult is a resilient, well developed creature, as a rule, with good internal arrangements for resisting shock and effecting repairs. The latter group of the two mentioned above-the very old-have a high mortality for two reasons: the process of ageing lessens their resilience and poor nutrition, possibly coupled with other illnesses prior to the burn, depresses their general health still further. For example, an elderly lady living in a room alone and just having recovered from pneumonia, subsisting mainly on cups of tea and bread and butter, accidentally sets fire to her clothing-it is not surprising that this patient succumbs to a burn that a young adult could endure with reasonable hope of survival. The previous health of the patient is too often ignored in assessing a burn.

4. The Site of the burn frequently affects the severity of the shock. For a given area burns of the face, head and neck, breasts, in women, genitalia and perineum in both sexes, are much more shocking than burns of other parts of the body. Possibly the profusion of sensory nerve endings in these regions plays a part.

5. The Agents (i.e. fire, water, steam, hot oil, acids, boiling porridge, various chemicals) may be of various temperatures and remain in contact with the skin for periods related to physical factors. For example a hand may be pulled away from a jet of steam from a kettle so that the burning process stops almost at once, boiling water takes longer to shake off hot oils and acids are even more tenacious. The clinging properties of the boiling porridge are dreaded by all who have treated burns in non-European children.

\section{Management of Burnt Patient.}

Far better than the use of the phrase "the treatment of a burn" is "the management of the burnt patient" and the first step in the case of a severe burn is to cover the burn area with anything that is sterile that will not stick-usually tulle gras in a single layer followed by thick layers of gauze and frrm crepe bandages-from then on for some considerable time, we forget the burn and treat the patient. (The same principles apply in the First Aid treatment of a severe burnavoid oils and creams, cover the burn with anything clean and treat the patient.) $A$ rapid assessment during this first dressing gives one some idea of the extent of the burn and a less accurate idea of the depth of the burn. Many classifications of degrees of burning may be found in the literature. Some authors have used six and even nine degrees. The modern approach is to use a less complicated scheme referring to three or even two degrees:-

\section{Degree.}

1. Erythema-redness-like sunburn

2. Blisters (Vesication)

3. Full thickness of skin and deeper

e.g. fat, muscle, bone as well.

\section{$\{$ SUPERFICIAL $\{$ DEEP}

The important decision is whether or not the skin has been destroyed in its full thickness-once this decision can be definitely made one can begin to plan for skin replacement (which will be needed in all full thickness losses-except for very small areas) or one can continue treatment with the relieving knowledge that control of shock will almost certainly be followed by fairly rapid recovery. The snare that lies in all this is that it is impossible to assess accurately the depth of tissue destruction in a severe burn during the first one or two days after burning; the erythema may go on-to blistering, the blisters may have unseen floors composed of full thickness damage. All areas may suffer so many changes long after the burning agent has been removed, that skin whose vitality is in the balance soon after burning may die during the subsequent forty-eight hours or recover and liveneeding no replacement. This state of affairs cannot be accurately foretold in a severe fresh burn.

Assessment of the area of a burn can usually be estimated by more than one method, a classical set of figures has been worked out by Berkow:-

As a rough general guide, all burns of greater area than $10 \%$ of the body surface will probably require some intravenous fluid to restore to normal the sudden and dangerous disturbance of the body's circulating volume.

In extensive burns this restoration of an equilibrium is a life saving measure--if the profound shock produced is not 
correctly stabilised within about two days, then the patient's creally in danger. Should this critical period be surlife is toes not mean that all is well-death may occur 列 toxaemia or from overwhelming infection invading the dead dying skin and fat. Even after this period the prolonged ard on the patient's resistance by a chronic infection, anaemia and protein loss associated with a large raw and and the failure to eat adequately without intensive energetic nursing aid, may still be fatal.

The first three months after burning may cover a series f severe illnesses. Once shock and infection are controlled the major life-saving measure that remains is the early, complete covering of all areas of full thickness skin loss by skin grafting.

A common question is "when should a burn be grafted?" The answer is "when it is ready"-ideally this is two or three weeks after burning, when by repeated dressing or bathing and actice removal of sloughs, all burnt tissue is removed and the raw area is smooth and red-a clean expanse of velvety granulation tissue.

\section{Physiotherapy}

Another common question is "when should physiotherapy start?" Once again tha answer is rather unhelpful-"when the patient is ready for it." In practice this means as soon as shock is controlled. Hands are either lightly splintered or dressings arranged so that they assume a position of function. Foot drop is guarded against. The patient is encouraged to move about in bed and take an actice part in feeding and any other reasonable activities. Breathing exercises may be of considerable importance.

ldeally a physiotherapist should be present when dressings are done or baths given, using these opportunities to try and maintain a full range of movement of all joints or as many as possible.

It is of vital importance to treat the patient and not the disease. Morale is often low, constant optimism is important - stressing progress at each dressing or bath. A commom fault to be avoided in reviewing the late established deformaties of severe burns is the dogmatic condemnation of those who treated the patient, and the assertion that skin grafting and "splinting" or often "splinting" alone would have prevented the deformity and, presumably, resulted in better residual function.

It is important to remind ourselves again and again that medicine has little room for "always" or "never". Sometimes, in superficial burns, puzzling and serious' residual impairment of function appears. Sometimes, in severe burns, function is astonishingly good when little care has been taken with grafting and maintaining functional positions of limbs and their joints.

General truths which far more nearly fit the facts are that:1. Full thickness burns result in an area of complete denudation of skin-proved when slough separates

2. Nature heals or attempts to heal such an area by a process which resembles the drawing together of the edges of a wound regardless of functional limitations which may be produced. 3 . The way in which the contraction of a raw area influences the part in which it lies, depends on:

(a) whether it is over a joint surface-if this os so the joint range becomes limited by rigid scar tissue formation. Changes in subcutaneous tissues, muscles, tendons and joint capsulr are largely secondary-rarely are tendons, muscles and the joint itself damaged by burning.

(b) whether the area is grafted early, so defying the edges to come together. Early skin grafting has the inestimable value of turning an open wound, with all its dangers of infection, protein loss and anaemia, into a closed wound. A closed wound offers time to think and plan, to gain maximum movement by physiotherapy, and prevents the laying down of further scar tissue.

No one with a reasonably wide experience of treatment of burns, claims that grafting entirely prevents contractures, but it minimises their severity beyond all measure, so that re-education and future reconstructive surgery can be undertaken earlier, and will give better results.

Conclusion.

Finally, I would say to all physiotherapists, beware of the unwanted guest who outstays his welcome-the longer you leave him the more difficult he is to eliminate-in other words - war on oedema-if left static it undergoes fibrous replacement, locking surfaces to the depths.

We cannot succeed in isolation-the patient needs help from all of us.

\section{PHYSIOTHERAPY TREATMENT}

These notes on physiotherapeutic measures used in the management of a burnt patient are intended only as a broad outline for possible treatment. As Mr. Walker has pointed out, physiotherapy starts "when the patient is ready for it" and as soon as shock is controlled.

\section{Pre-operative Treatment.}

(a) Breathing exercises.

(b) A full range of movement to all joints not directly involved in the burnt area, and as much as possible without pain to involved joints.

(c) Badly burnt patients are given static contractions to all parts before grafting.

(d) Where the burn is over the joint, static contractions to the muscles around the joint should be given. Let pain be the limiting factor in joint .movements in a fresh burn.

(e) Elderly patients should be encouraged to sit up in a chair for part of the day.

(f) Children with severe burns are prone to broncho-pneumonia. Therefore postural drainage is usually given automatically to every case. This can be done by either tipping the bed or elevating the child on pillows. If he is allowed to blow bubbles, or for those physiotherapists who have strong nerves, - a trumpet or whistle, he will tolerate this position for a long time.

Post-operative Treatment.

Move everything but the grafted area for the first few days, or until permission is received from the surgeon to move the grafted area.

\section{Donor Areas.}

Static contractions to the muscles lying beneath the raw area. This is very important especially if the graft has been taken from the thighs. Aim to get straight leg raising as soon as possible.

Dressings.

A careless dressing technique can allow infection to creep into the grafted area with resultant necrosis.

The vascular supply to the wound can be seriously embarrassed by a bandage too tightly applied, with the same result as above.

When the dressings are removed all grafted parts should be moved, especially where the graft is over a joint. Range should be increased very gradually to avoid displacing or damaging the graft.

Baths.

\section{(a) Saline}

Normal saline baths may be given at an early stage and are especially useful where burning is extensive. Movements are carried out in the bath, and the separation of sloughessential before grafting-is hastened.

(b) $L u x$.

Lux helps to steralize the burnt area ( 2 small packets to a full bath). 
If their is a small hair drier available, use this to dry the raw areas rather than a towel, which may cause further bleeding.

Splints.

These are usually made of padded Kramer wire or light Plaster of Paris shells-thinly padded. If the graft is over a flexor surface-particularly the knee, elbow and wrist joints a splint is worn to minimise contractures. Splints should be worn at night and removed for exercises.

\section{Grafts over the Groin.}

The patient is nursed in lying or prone lying. He may be allowed to stand or walk, but sitting should be discouraged. For children, hold the legs in a good position by means of towels and sand bags.

Grafts over the Neck.

Nursed in lying with one very soft pillow.

Grafts over the Elbow and Knee (Extensor Surfaces.) -

Encourage extension not flexion.

Grafts in Lower Leg and Foot.

1. Quadriceps drill only for four days.

2. 5th day. Movements to toes and ankles.

3. 6th to 10th day. Hang the leg over the side of the bed for only 5 minutes or less every half hour. Remain with the patient and watch the graft carefully. If the graft goes too blue replace in bed immediately. In general the hazards of cyanosis-indicating a poor venous return from a free graft or flap--are much greater than those of poor arterial supply -indicated by pallor of the graft or flap.

4. 7 th to 14th day. Using a crêpe bandage, bandage the leg from the toes to the tubercle of the tibia and sit the patient out in a chair for half an hour twice daily.

5. 8 th to 20th day. Walking. Watch the graft. If it becomes very blue return the patient to bed immediately.

All times are approximate and must be modified by the type of graft.

Grafts to Hands.

These are bandaged in a functional position until the graft has taken. No movements are given. Care should be taken to see that the hand is elevated and maintained in this position to prevent swelling. Finger tips must be exposed whenever possible.

\section{Grafts over Concave Surfaces.}

No movements are given as it is thought that exercises increase the tendency for fibrous tissue to contract and increase the risk of haematoma by disturbing pressure dressings or mould, holding the graft in position against the walls of the cavity.

\section{Oedema.}

Explain the dangers of oedema and leave the patient to work it away. Encourage any active movements and give the patient plasticine or a small rubber ball to squeeze. Where movement is too small to be useful, if the limb is in plaster, hang it up and let gravity drain it away.

No passive movements should be given unless by a doctor under an anaesthetic, or when specially ordered and the graft is "safe" and maturing.

\section{Massage.}

Very little is gained by massage in the early stages. When the wound is completely healed, massage with olive oil will help to soften the grafted area, which is often rather dry or scaly.

\section{Wax Baths.}

Very cool wax baths are also helpful. The danger of sepsis is negligible and these can be given after about three weeks.

\section{Short Wave Diathermy.}

Unsatisfactory because of the local difficulty of preventing over-heating, which may cause ulceration. The healing is patchy and may cause an epithelioma after years of chronic ulceration.

\section{Infra Red.}

Where there is gross circulatory disturbance, for example in a "frozen" hand, reflex dilation in an infra red tunnel may be given. This method was used with success during the war, when immediate surgery was impractical, owing to lack of space in base hospitals.

\section{Ultra Violet Ray.}

Hazardous on a free graft as there is a danger of pigmentation and sometimes. ulceration. This is usually a diffuse yellowish pigment which does not fade and should be guarded against, especially where the graft is on the face.

You will not be loved by your patient if he acquires a beautiful tan in the summer but is left to face the winter with a bright yellow patch on his face!

In estimating the area of an irregularly shaped burnt area it is sometimes useful to remember that the palm of the hand is about $1 \%$ of the body surface.

$$
\begin{aligned}
& 8 \% \text { Head and Neck } \\
& 16 \% \text { Upper limbs } \\
& 38 \% \text { Trunk-back and front } \\
& 38 \% \text { Lower Limbs }
\end{aligned}
$$

Wallace has simplified this by introducing a scheme based on the magic number 9 .

\section{Deliverance}

from pain ... the humanitarian function of of the practitioner.... now accomplished with greater speed, more economy and with a higher degree of safety than ever before.

Full details and elinical notes on the use of

\section{$\mathrm{R} \overline{\mathrm{ENO} O \bar{T} \mathrm{~N}}$}

in the treatment of Migraine, Neuritis, Lumbago, Sprains and Myalgia may be obtained from

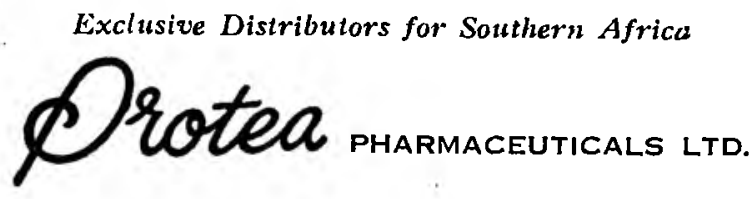

P.O. BOX 7793 JOHANNESBURG 